\title{
Accuracy of lung cancer staging in the multidisciplinary team setting
}

\author{
Chong-Kin Liam', Yong-Sheng Liam², Mau-Ern Poh ${ }^{1}$, Chee-Kuan Wong ${ }^{1}$ \\ ${ }^{1}$ Department of Medicine, Faculty of Medicine, University of Malaya, Kuala Lumpur, Malaysia; ${ }^{2}$ Clinical Investigation Centre, University Malaya \\ Medical Centre, Kuala Lumpur, Malaysia \\ Contributions: (I) Conception and design: All authors; (II) Administrative support: YS Liam; (III) Provision of study materials or patients: YS Liam, \\ CK Liam; (IV) Collection and assembly of data: YS Liam, CK Liam; (V) Data analysis and interpretation: None; (VI) Manuscript writing: All \\ authors; (VII) Final approval of manuscript: All authors. \\ Correspondence to: Chong-Kin Liam. Department of Medicine, Faculty of Medicine, University of Malaya, 50603 Kuala Lumpur, Malaysia. \\ Email: liamck@ummc.edu.my.
}

\begin{abstract}
Accurate staging of lung cancer is of utmost importance in determining the stage-appropriate treatment and prognosis. Imaging tests which include contrast-enhanced computed tomography (CT) examination of the chest to include the liver and adrenal glands and 18-fluoro-2 deoxyglucose positron emission tomography (PET)/CT scan facilitate the initial tumor node metastasis (TNM) staging of the disease and provide guidance on the optimal biopsy site and biopsy method. The diagnostic and staging approach should be tailored to the individual patient according to risk, benefit, patient preferences, and available expertise. Diagnosis and staging should preferably be accomplished with a single procedure or the least number of invasive procedures if more than one is needed. Ideally, centers managing lung cancer patients should have a multidisciplinary thoracic oncology board prescribing personalized evidence-based management tailored to each individual patient. Multidisciplinary team (MDT) meetings provide a platform for key experts from various disciplines to contribute specific advice on the management of each individual patient. As assessment of mediastinal lymph node involvement is an important component of lung cancer staging, optimal mediastinal staging can be achieved with a variety of techniques that can be discussed and performed by the various specialists in the MDT. Despite a relative paucity of quality evidence that MDT contributes to improvements in lung cancer survival outcomes, this approach has evolved to become the standard of care in many centers around the world. Thoracic MDT has resulted in more focused and timely investigations for histopathologic diagnosis and disease staging which translate into earlier treatment initiation. Moreover, there is increasing evidence that MDT care facilitates and allows access to investigations that lead to improved accuracy of tumor and nodal staging. However, there is still a paucity of evidence on the accuracy of lung cancer staging in the MDT setting.
\end{abstract}

Keywords: Accuracy; lung cancer; multidisciplinary; staging

Submitted Aug 04, 2019. Accepted for publication Nov 18, 2019.

doi: $10.21037 /$ tlcr.2019.11.28

View this article at: http://dx.doi.org/10.21037/tlcr.2019.11.28

\section{Introduction}

Globally, lung cancer causes the most deaths from cancer in both men and women (1). Approximately $85 \%$ of lung cancer cases are non-small cell lung cancer (NSCLC) while small cell lung cancer (SCLC) accounts for most of the rest. The majority of patients present for a diagnostic workup because of lung cancer-related symptoms or because of incidental findings on chest radiographs or computed tomography (CT) scans. The aim of the initial assessment is to acquire a thorough clinical and radiologic information to direct tissue biopsy and staging which will then determine the appropriate stage-specific treatment. This approach applies both to patients suspected of having NSCLC or SCLC. 


\section{Initial assessment of patients suspected of having lung cancer}

The aim of the initial work-up of a patient suspected of having lung cancer is a timely diagnosis and accurate staging in order to recommend stage-appropriate therapy. The approach to such a patient is dependent on the clinical presentation and the technical expertise at the practicing institution. Patient goals and individual preferences should also be assessed and established to allow shared decisionmaking regarding diagnostic and treatment options. These may vary from invasive investigations with a curative intent to settling for symptomatic treatment only with minimal or no investigation.

At the initial assessment, a symptom-driven assessment will guide the physician towards the appropriate imaging to identify nodal involvement or distant metastases. A metaanalysis of 25 studies assessing the clinical evaluation in detecting extrathoracic metastases observed high negative predictive values of $95 \%$ for central nervous system, $94 \%$ for intra-abdominal, and $89 \%$ for osseous spreads (2). In addition, the presence or absence of symptoms can be prognostic. Patients who are asymptomatic or have symptoms only related to the primary tumor have a better prognosis compared to patients who are symptomatic of metastatic disease as demonstrated by a cohort of 1266 patients (3). The likelihood of metastatic disease can be predicted based on a thorough physical examination together with the appropriate laboratory investigation, especially in NSCLC (2). Abnormal laboratory test results can direct further investigations and imaging to guide diagnostic and staging work-up.

Imaging is an essential initial step to assess the clinical extent of disease in the lung, the mediastinal and hilar lymph nodes, and distant metastases as well as to identify an optimal site and modality for tissue biopsy to establish the histopathological diagnosis and also the disease stage. Whenever feasible and contingent upon accessibility and risk involved with the procedures, the diagnosis and disease stage should be determined simultaneously by selecting for biopsy the lesion that establishes the highest stage of the disease (4). Biopsy of a lower-stage lesion will often require additional procedures and biopsies to more accurately stage the disease. For example, thoracentesis for pleural fluid cytology examination for M1a disease, transbronchial needle aspiration (TBNA) biopsy of mediastinal N2 or N3 lymph nodes or biopsy of a suspected metastasis (M1b or M1c) may establish both the diagnosis and stage of the disease (5). If pleural fluid for cytology is negative for malignant cells, image-guided needle biopsy of the pleura or pleuroscopy should be performed to confirm pleural metastasis. However, some patients may require more than one imaging study and/or tissue sampling procedures for both diagnosis and staging. If imaging reveals multiple sites of metastases, selecting the safest or easiest approach for pathologic confirmation of lung cancer is preferred. Unnecessary additional procedures can be avoided by careful study of imaging findings.

Although diagnosis and staging are usually separately discussed, they are integrated into a single process in clinical practice where prior to performing a biopsy for diagnosis, staging and the potential for treatment are considered at the same time. Hence, assessments of the patient's underlying pulmonary physiology, comorbidities if any and ability to tolerate treatment, and the initial imaging are done in parallel. The choice of biopsy site and procedure used are influenced by tumor location and characteristics, patient comorbidities, availability of equipment, local expertise and procedural proficiency, as well as patient preferences $(6,7)$.

\section{The role of multidisciplinary team (MDT) in the assessment of patients suspected to have lung cancer}

Changes of the diagnostic, staging and therapeutic landscape in lung cancer in recent years necessitate a multidisciplinary approach in managing the disease as is the case in other cancers (8). The best in lung cancer care requires a MDT of experts who regularly manage lung cancer patients. The pulmonologist plays a central role in the MDT starting with making the diagnosis, staging and eventually management including end-of-life care. Optimal treatment also requires care which must be prompt and patient-centered. Close collaborations between pulmonologists and radiologists, nuclear medicine specialists, pathologists, thoracic surgeons with lung cancer specialization, medical and radiation oncologists, palliative care physicians, specialist nurses and pharmacists form the basis of a MDT. To coordinate patient care among these various disciplines, multidisciplinary lung cancer clinics are recommended (8). With the shift towards a multidisciplinary approach, many institutions offering cancer care incorporate a MDT for case discussion, collaborative decision-making, sharing of knowledge among the team members and for making collective recommendations for the diagnosis, staging and appropriate treatment of patients with lung cancer which are evidence- 
based (8-16).

Involving an MDT early can expedite the diagnosis and staging processes with fewer unnecessary tests. Regular MDT meetings have become a norm in oncology and have attained the status of being the prime decisionmaking platform for managing patients with cancers. Such multidisciplinary discussions are held either in multidisciplinary clinics and/or tumor boards. While differing terminology such as multidisciplinary clinics, multidisciplinary cancer conferences, multidisciplinary case reviews, or tumor boards are used to reflect the differences in the structure of the organization, these MDT meetings all provide a platform for the various disciplines to convene on a regular basis to deliberate on the diagnosis, staging and treatment of lung cancer patients. These MDT meetings serve to ensure timely diagnosis and the best available evidence-based care is given to patients. They also serve to facilitate effective communication and information among all health care professionals to keep its members abreast on the latest developments in thoracic oncology, while providing medical education to trainee doctors. The MDT approach allows monitoring of adherence to evidencebased guidelines while streamlining resources to improve efficiency and cost effectiveness, and shortening waiting times.

An MDT setting facilitates and eases communications and face-to-face discussion among the healthcare professionals involved with the management of the patient. A team effort is needed to ensure that the most appropriate tests are selected, procedures are safe and performed correctly so that the results obtained can be used to positively affect the management of the patient. Each institution can develop their own algorithm for tissue sampling for the diagnosis and staging of NSCLC by adapting guidelines of the American College of Chest Physicians (ACCP), European Society of Thoracic Surgeons (ESTS) and International Association for the Study of Lung Cancer/American Thoracic Society (IASLC)/European Respiratory Society (ERS) $(11,14,17,18)$. These guidelines have a common goal to expedite investigations, optimize the utilization of available resources, standardize the approach to evaluation and treatment of patients with lung cancer and in general enable a more efficient delivery of lung cancer management service $(4,11)$. A coordinated effort and good communication between the bronchoscopist, thoracic surgeon, or interventional radiologist on one hand and the cyto-/histopathologist on the other to collect sufficient specimens for diagnostic testing is of utmost importance especially when the diagnosis and staging hinge on the yield of these procedures. Furthermore in this era of personalized treatment of advanced NSCLC based on histologic subtyping and molecular profiling, adequate specimens should be obtained and processed in a way that provides enough information to guide therapy without wastage of tissues. As many diagnostic tests may be required the specimen obtained should be used sparingly at each step $(13,19)$ Pathologists must conserve tissue during diagnostic examination by using a limit panel of immunohistochemistry for histologic subtyping of NSCLC and prioritize molecular testing for targeted therapies in advanced NSCLC (20-22).

In the initial evaluation of the disease, pulmonologists play an important role in performing diagnostic procedures such as bronchoscopy, endobronchial ultrasound (EBUS), thoracentesis, and pleuroscopy to sample tissue specimens to establish the diagnosis and stage as well as guide tissuedirected and personalized targeted treatment $(23,24)$. Moreover, in European and many Asian multidisciplinary centers, pulmonologists direct the MDT meetings and are the ones responsible for the prescription of targeted therapy and cytotoxic chemotherapy to patients with lung cancer $(15,25)$. In addition to monitoring patients with resected stage II and III NSCLC, adjuvant chemotherapy is also prescribed by the pulmonologist in many of these centers. Adjuvant chemotherapy is also considered on a case-to-case basis in patients with resected stage IB disease and a primary tumor measuring more than $4 \mathrm{~cm}$ (26). The patient's underlying comorbidity, time since surgery and postsurgical recovery have to be factored in when deciding on adjuvant chemotherapy in an MDT meeting (26).

\section{Timeliness in the evaluation of patients suspected to have lung cancer}

The evaluation of patients suspected to have lung cancer should be performed in the shortest time possible and in the most efficient manner (18). As mentioned, the initial evaluation to make a diagnosis, to determine the clinical stage of the lung cancer and the development of a treatment plan, often occurs concurrently and not necessarily in a sequential manner. While the majority of patients can be investigated as outpatients, patients with comorbidities or complications such as respiratory failure, severe hemoptysis, disabling brain or bone metastases may warrant hospitalization and work-up as in-patients. The diagnosis should be established expeditiously especially when SCLC 
is suspected and in patients with large, rapidly growing central tumors or bulky multi-station mediastinal nodes which may compromise the airways. Some observational studies have shown a shortened time to treatment initiation, improved surgical resection rates and patient satisfaction with multidisciplinary thoracic oncology team review and healthcare facility-implemented rapid diagnostic and investigation processes (27-29). However, other patientrelevant outcomes such as survival have not been shown to improve despite such interventions. Preferably the initial evaluation should be completed within six weeks in patients with tolerable symptoms and no obvious complications (18). While the growth of most cases of NSCLC is relatively slow with a volume doubling time of 3 to 6 months, rapid disease progression can sometimes occur during the evaluation period. Since disease progression can occur with treatment delay, diagnosis, staging, and treatment initiation should be expedited. In a case series, progression of disease was documented in $13 \%, 31 \%$, and $46 \%$ of patients by CT and PET scan at 4,8 , and 16 weeks from the first imaging, respectively, with new distant metastasis document in $3 \%, 13 \%$, and $13 \%$ of cases after 4,8 , and 16 weeks, respectively (30). Restaging investigations should therefore be strongly considered after 4 to 8 weeks of delay (30). Staging CT and PET/CT should be done not more than 60 days before surgery (4).

\section{The importance of accurate staging in lung cancer}

Accurate staging to determine the extent of the disease is of great importance in patients diagnosed with lung carcinoma because of its impact on treatment options and prognosis $(4,17,26)$. The types of staging in patients with NSCLC include clinical staging, surgical-pathologic staging and restaging for recurrent disease. The clinical-diagnostic stage, assigned the prefix c, is based on information from clinical examination, and all laboratory, radiologic, and pathologic investigations performed before surgical resection (5). The accuracy of clinical-diagnostic staging is dependent on the intensity of the preoperative assessment; an insufficiently investigated patient may be inaccurately under- or over-staged. An incorrect or insufficient cTNM classification can lead to erroneous decision on treatment options, such as offering palliative treatment to potentially curable patients or conversely subjecting patients with advanced disease to aggressive curative treatment. The likelihood of erroneous staging and treatment decision is reduced by MDT reviews. While clinical evaluation and imaging such as CT provide a basis to estimate the extent of the disease, additional tests are needed in most cases for accurate staging. The more accurate surgical-pathologic stage (pTNM) is based on the clinical stage (cTNM) plus additional histopathologic information from the resected tumor and lymph nodes (5). This staging confirms the T descriptor, $\mathrm{N}$ descriptor, and histologic type of the lung cancer. In addition, it incorporates information on the histologic grade, resection margins, and presence of any lymphovascular infiltration. The pTNM stage is considered to be the 'true' stage, and the degree of concordance between the cTNM and pTNM stage is a reflection of the diligence and quality of the evaluation to make the diagnosis and to stage the tumor. A stage for retreatment is assigned if there is disease recurrence with a whole new set of staging evaluations followed by new treatment recommendation.

In patients who undergo surgical resection, all staging parameters recommended by the American Joint Committee on Cancer (AJCC) have to be carefully examined by the histopathologist. The size of the tumor, extent of invasion, clearance of surgical margins, and the presence of regional lymph node metastases should be determined by a thorough examination of the resected specimens. The number of affected lymph node stations should be accurately documented since this will affect the surgical-pathologic stage, the prognosis, and the need for adjuvant treatment. Even in patients undergoing a sublobar resection, N1 and N2 lymph node stations should be sampled or resected unless technically not feasible or associated with increased operative risk (4). For lobar resection, the lobe should be extensively dissected and examined for any lymph node involvement.

\section{Lung cancer staging procedures}

Lung cancer staging can be achieved by several noninvasive and invasive investigations. The selection of the most appropriate investigation by the MDT should take into account of the way the patient presents and also the patient's preference. The algorithm for staging should include a contrast CT of the chest and upper abdomen to include the liver and adrenal glands, positron emission tomography (PET)/CT to guide mediastinal staging and to exclude extrathoracic metastases in patients considered for curative surgery, endosonography-guided needle sampling of mediastinal lymph nodes, and brain imaging as clinically indicated (25). A PET/CT is recommended in patients who 
are potential candidates for therapy with a curative intent (clinical Stage IA-IIIB) (8).

\section{Imaging modalities for accurate staging of lung cancer}

Imaging modalities for accurate lung cancer include a CT, whole body 18-fluorodeoxyglucose (FDG) PET or an integrated PET/CT, and magnetic resonance imaging (MRI) of the brain. CT is the most widely-used imaging modality for staging across many different centers because it is readily available and relatively inexpensive. Patients suspected of having lung cancer eligible for treatment should undergo at least a contrast enhanced chest CT with imaging through the upper abdomen, liver, and adrenal glands $(17,26)$. Chest CT provides the basis for initial assessment of the tumor, node, and metastasis (TNM) stage of the disease.

While CT of the thorax is able to identify the location of the tumor and the presence of involved mediastinal lymph nodes, it is limited in its ability to differentiate benign from malignant lung nodules and enlarged lymph nodes. The sensitivity and specificity of CT scan in detecting malignant mediastinal lymph node involvement are relatively low at $55 \%$ and $81 \%$, respectively (17).

In contrast, FDG-PET scanning has a much higher accuracy in identifying metastasis to the mediastinal lymph nodes. In a systematic review of 45 studies, the sensitivity, specificity, positive predictive and negative predictive values of PET-CT was $80 \%, 88 \%, 75 \%$ and $91 \%$, respectively (17). PET-CT was also more sensitive in detecting distant extrathoracic metastases except for brain metastases which requires imaging with contrasted brain CT or MRI (31-37). FDG-PET is also limited in its ability to differentiate benign (e.g., inflammation or infection) from malignant disease. Thus, tissue sampling of suspicious lesions is still mandatory.

As mentioned above, the high negative predictive value of FDG-PET pre-empts more invasive diagnostic investigations if there is no extra FDG-uptake in the mediastinum, except in special circumstances where the tumor is located centrally, or in the case of adenocarcinomain-situ and N1 disease. This allows curative surgery to be undertaken with confidence.

However, the high false-positive rate of PET-CT means a positive FDG-PET examination necessitates histo-/cytopathological confirmation. PET-CT also has a significant false-negative rate in certain circumstances such as when lymph nodes are only moderately enlarged or not at all (38). In these circumstances, metastases in nonFDG-avid normal-sized or moderately enlarged lymph nodes should be excluded through diagnostic procedures such as EBUS or mediastinoscopy $(39,40)$. Approximately $4 \%$ of patients with what appears to be stage I disease and a normal PET-CT have been found subsequently to have N2 disease. Since metastases can occur in non-FDG-avid normal sized or moderately enlarged lymph nodes, there is no doubt that larger non-FDG-avid lymph nodes with a higher probability of N2 disease should be sampled. N2 lymph nodes which are positive for metastases increase the likelihood of positive N3 nodes. Therefore, it is advisable that evaluation of the mediastinum includes sampling of the subcarinal and contralateral lymph node stations (4).

The specificity of PET-CT is affected by the prevalence of other FDG-avid diseases in the population such as tuberculosis (TB) and sarcoidosis and has been reported to be not more than $90 \%$ in such populations (17). In countries where TB is endemic, the accuracy of PET/CT in lymph node staging may be lesser. In a meta-analysis of seven studies evaluating the diagnostic performance of PET/ CT for lymph node staging in NSCLC patients located in regions endemic for $\mathrm{TB}$ versus regions not endemic for $\mathrm{TB}$, the pooled sensitivity, specificity, positive likelihood ratio and negative likelihood ratio were 66\%, 92.7\%, 5.86\% and $0.41 \%$, respectively for patient-based analyses, while these were $59.4 \%, 96.5 \%, 9.37 \%$ and $0.31 \%$, respectively for lesion-based analyses (41). TB endemic regions have lower sensitivity and similar specificity as non-TB endemic regions.

The National Comprehensive Cancer Network guidelines recommend PET scanning even in peripheral radiographic stage IA1-3 (T1a-cN0M0) NSCLC (4) even though metastatic disease is infrequent with a prevalence of about 4\% (42). While surgical resection may proceed in this situation without prior PET scanning and with mediastinal sampling performed intraoperatively, preoperative PET scanning should be performed in patients of high surgical risk to exclude them from unnecessary surgery $(39,40,42-45)$.

Except for brain metastases, whole body PET or PET/ CT is more accurate than CT for detecting pleural and extrathoracic metastases (31-37). According to small randomized studies and case series, PET or PET/ CT scanning can discover unsuspected metastases in $6 \%$ to $36 \%$ of NSCLC patients (46-49). In addition, alteration of the TNM staging occurs in up to $22 \%$ of patients following 
PET or PET/CT $(32,37,50,51)$. Although randomized trials showed that although integrated PET/CT prevented unneeded futile thoracotomies in $7 \%$ to $17 \%$ of patients with potentially resectable NSCLC by altering the TNM staging $(52,53)$, survival improvement has not been demonstrated.

Gadolinium-enhanced MRI of the brain is more sensitive than non-enhanced MRI or CT in detecting brain metastases $(54,55)$ Gadolinium-enhanced brain MRI is used to exclude brain metastases in patients with early stage disease planned for curative surgery, to investigate patients symptomatic of brain metastases and to assist in treatment decision making in asymptomatic patients with locally advanced or advanced NSCLC.

\section{Choice of modality for mediastinal sampling}

As mentioned earlier, assessment of the mediastinal lymph nodes is an important component of lung cancer staging. Absence of mediastinal lymph node involvement is an important determinant of tumor resectability. Pathologic confirmation of tumor involvement of enlarged mediastinal lymph nodes on CT or metabolically active nodes on PET scan is recommended in patients with otherwise potentially resectable tumor. International guidelines recommend selecting needle-based or surgical techniques for obtaining a cytohistologic diagnosis from the primary tumor and/ or mediastinal or hilar lymph nodes (8). Selection of the optimal biopsy site and sampling modality should be driven by evidence-based protocols that involve a lung cancer tumor board or MDT. With a wide variety of possible techniques that can be performed by the various specialists in the MDT, the choice of the modality for optimal mediastinal lymph node staging is a collective decision made by the MDT.

Having a good working knowledge of the TNM staging system, the mediastinal lymph node stations (56) and treatment options for each stage of NSCLC, is essential for decision making regarding the optimal biopsy site. Assessing the risk of N2 and N3 lymph nodal involvement and the risk of metastatic disease is important when selecting specimen sampling procedures. The choice of a tissue sampling procedure for patients with suspected mediastinal nodal involvement is dependent on the site of the primary tumor and suspected lymph nodes, available expertise, treatment options for the suspected stage, and patient safety and preferences (25). When choosing one modality over another, knowledge of the accessibility of the primary tumor and mediastinal nodes by the variety of minimally invasive and invasive techniques is essential. Needless to say, the modality that is safest to perform and has the highest yield to achieve a diagnosis should be chosen. Transbronchial needle aspiration guided by endobronchial ultrasound (EBUS-TBNA) with or without transesophageal endoscopic ultrasound fine needle aspiration (EUS-FNA) are the preferred initial procedures for sampling suspected nodal metastasis(es).

\section{Minimally invasive procedures for lung cancer staging}

Blind TBNA which has a low diagnostic yield of approximately $40 \%$ when used to sample suspicious lesions or enlarged mediastinal lymph nodes noted on CT has been superseded by endoscopic-ultrasound guided procedures, EBUS and EUS with diagnostic yields up to 90\%. While EBUS-TBNA enables real-time sampling of the paratracheal, retrotracheal, subcarinal, hilar, and interlobar lymph nodes (stations 2R, 2L, 3p, 4R, 4L, 7, 10R, 10L, 11R, and 11L) (56), EUS- FNA allows sampling of the mediastinal nodes situated next to the esophagus which include the retrotracheal (station $3 \mathrm{p}$ ), left lower paratracheal (station 4L), aortopulmonary (station 5), subcarinal (station 7), and the paraesophageal and pulmonary ligament (stations 8 and 9) nodes in the inferior mediastinum (56) as well as liver and left adrenal metastases. A more complete mediastinal staging can be accomplished by combining EBUS-TBNA and EUS-FNA if needed as the complementary reach of these two procedures allow assessment of different parts of the mediastinum. EBUSTBNA, EUS-FNA and combined EBUS/EUS-NA have sensitivities of about $89 \%, 89 \%$ and $91 \%$, respectively. These minimally invasive sampling techniques are as accurate as mediastinoscopy in experienced hands $(57,58)$, thus reducing the need for surgical staging. Institutions that perform higher volumes of procedures may be more willing to sample smaller and difficult to reach lesions.

Rapid on site cytopathology examination (ROSE) during EBUS-TBNA improves diagnostic yield of EBUS-TBNA, shortens the time of the procedure, and improves adequacy of specimen for molecular testing but according to the World Association for Bronchology and Interventional Pulmonology guidelines ROSE is not necessary for every case for an experienced operator (59). The accuracy of these minimally invasive EUS-guided needle sampling procedures should be weighed in the context of its limitations including 
the inability of EBUS-TBNA to access nodes in the inferior mediastinum (stations 8 and 9) and aortic nodes (stations 5 and 6) and variable operator proficiency. The usage of EBUS-TBNA, EUS-NA or EBUS/EUS-NA for lung cancer staging reflects the need for a multidisciplinary coordinated effort such as between the pulmonologist and the cytopathologist to collect and triage specimens. Both the bronchoscopist and pathologist must understand the rationale for histologic and molecular testing of tumor specimens and ensure that sufficient cytologic specimens can be processed in a specimen conserving manner without wastage to guide treatment in the current era of personalized targeted therapy.

Invasive mediastinal lymph node sampling is recommended in patients with discretely enlarged mediastinal nodes with or without PET uptake and in the absence of distant metastases (17). Invasive mediastinal lymph node sampling is also recommended for patients with a normal mediastinum on CT scan or PET/CT if the primary tumor is centrally located or if there is hilar lymph node enlargement (17). However, when there is overwhelming evidence of extensive tumor infiltration or distant metastases, these invasive procedures to sample enlarged mediastinal nodes are not required (17). Preoperative mediastinal staging is also not necessary in patients with peripheral lesions not larger than $3 \mathrm{~cm}$ and non-hypermetabolic non-enlarged lymph nodes by CT/ PET, as the risk of N2 and N3 disease is low (17). These patients with suspected T1a(mi)N0M0, T1aN0M0, T1bN0M0, or T1cN0M0 (i.e., stage IA1/IA2/IA3) disease may proceed with surgery with intraoperative mediastinal lymph node systematic sampling or dissection rather than preoperative invasive mediastinal staging.

\section{Invasive surgical procedures for lung cancer staging}

If despite negative results from minimal invasive procedures described above, more invasive surgical staging by cervical mediastinoscopy (for stations 1, 2, 3, 4, anterior 7, 10), anterior mediastinotomy (or Chamberlain procedure) (for stations 5 and 6) or video-assisted thoracic surgery (VATS) for ipsilateral mediastinal nodes (stations 4, 5, 6, 7, 8, 9, 10 to 14 ) is recommended if suspicion of mediastinal node involvement remains high (17). In the special circumstance of left upper lobe tumors, assessment of the aortopulmonary window nodes using invasive procedures such as extended cervical mediastinoscopy, Chamberlain procedure or VATS maybe considered if involvement of other mediastinal node stations are not apparent on PET/CT scan (17).

For patients who undergo surgical resection, complete mediastinal lymph node dissection or at least systematic mediastinal lymph node sampling is needed to accurately assess the pathologic stage to determine the need for adjuvant therapy (8). Whether mediastinal lymphadenectomy confers a survival benefit over systematic mediastinal lymph node sampling is unclear, but lymphadenectomy is generally recommended if there is $\mathrm{N} 2$ node involvement. If incidental N2 disease (IIIA) is discovered at surgery for clinical stage I or II NSCLC despite thorough preoperative staging and provided that complete resection of the $\mathrm{N} 2$ nodes and the primary tumor is technically feasible, completion of the decided lung resection and mediastinal lymphadenectomy is proposed provided an $\mathrm{R} 0$ resection is deemed possible (8).

If discrete $\mathrm{N} 2$ nodal involvement is identified preoperatively (IIIA), the multimodality treatment plan of either definitive chemoradiation therapy or induction therapy followed by surgery should be decided upon after discussion by the MDT which should consist of at a least a thoracic surgeon, medical oncologist, and radiation oncologist (8). Multimodality treatment such as these should be carried out in centers with experienced MDTs to co-manage the potential toxicities and complications associated with the prescribed treatment.

The AJCC, Union for International Cancer Control (UICC), and IASLC recommend at least six lymph nodes are removed during surgery, i.e., a representative node from each station, three from N1 stations and three from N2 stations, one of which should be subcarinal node (station 7) for accurate staging $(5,60)$. Adequate surgical $\mathrm{N}$ staging should include systematic sampling or dissection of nodes from stations $2 \mathrm{R}$, $4 \mathrm{R}, 7,10 \mathrm{R}$ and $11 \mathrm{R}$ for right-sided tumors; stations 5, 6, 7,10L and $11 \mathrm{~L}$ for left-sided tumors; with station 9 nodes included for tumors of the lower lobes. Intrapulmonary lymph nodes at stations 12-14 are usually examined by the pathologist in lobectomy or pneumonectomy specimens but may be separately removed by the surgeon when sublobar resections are performed such as segmentectomy. The sampled or dissected nodes should be labelled according to the IASLC nodal definitions and chart (61).

Mediastinal lymphadenectomy involves the surgical resection of all mediastinal lymph node tissues identifiable based on anatomic landmarks (62). The accuracy of mediastinal staging is expected to be better with lymphadenectomy performed during surgery compared with 
nodal biopsy by minimally invasive or invasive procedures. If more limited mediastinal node sampling is performed and the result is negative, opinions are divided as whether N0 status should be recognized. While some would classify this as $\mathrm{pN} 0$, others would designate this as $\mathrm{pNO}$ (un) to reflect a degree of uncertainty.

The number of metastatic hilar or mediastinal node or lymph node station has survival impact. The international multispecialty Staging and Prognostic Factors Committee appointed by IASLC considered further subdivisions of the $\mathrm{N}$ categories of the TNM staging system to include the number of node stations involved. Analyses of the lung cancer database showed that there are differences in overall survival between p-stage tumors with single $v$ s. multiple $\mathrm{N} 1$ or $\mathrm{N} 2$ station involvement, but no difference in prognosis between multiple N1 station involvement (N1b) and a single-station mediastinal nodal skip metastasis without N1 involvement (N2a1) (63). This $\mathrm{N}$ subgrouping is not included in the eighth edition of the TNM stage classification, mainly because it cannot be accurately determined in clinical staging.

\section{The importance of high-volume specialized centers and MDTs}

Data show that survival in patients with lung cancer is improved when they are treated by high-volume specialized centers and MDTs which provide more complete staging, better adherence to evidence-based guidelines, and better treatment decisions than low-volume or nonmultidisciplinary centers $(8,64,65)$. The ERS/ESTS guidelines for fitness for radical therapy of lung cancer recommend the management of lung cancer patients by MDTs comprising pulmonologists, thoracic surgeons, medical oncologists, and radiation oncologists (66). The ACCP recommends the same for patients with potentially resectable lung cancer (8). Evidence supporting the MDT management model comes mostly from publications on cancer management in general and a few studies in lung cancer. A single center study in Scotland reported a significant improvement in the overall survival of patients with inoperable NSCLC from 3.2 months in 1997 to 6.6 months in 2001 which was attributed to the introduction of MDT management in the later year (65).

Despite a relative paucity of quality evidence that multidisciplinary care contributes to better survival outcomes, the MDT approach has evolved to become the standard of care in many centers around the world.
A systematic review of the evidence for multidisciplinary management of lung cancer has been published, reflecting the paucity of evidence for MDT practice in this disease (67). Assessing the effects of MDT care on the different outcomes of lung cancer such as accuracy in staging and survival improvement is difficult because of constraints in performing randomized trials due to the difficulty in recruiting patients where this practice is not the standard of care. Thus, studies have been mostly observational. In a review, Patkar et al. identified 15 studies which included various cancers and not limited to lung cancer, and comprising a single randomized controlled trial and mostly case series (68). These studies mostly studied patient outcomes before and after the implementation of MDT management. Interpreting the results of observation studies are made difficult because of the confounding effects of more effective therapies such as the tyrosinekinase inhibitors and other advances in treatment including minimally invasive surgical techniques that have been shown to improve the survival of lung cancer patients (69).

When it comes to accuracy of staging, MDT meetings do facilitate discussions between the various experts regarding the choice of site and technique used for tissue sampling to ensure that the site with the highest probability of obtaining tissue or the site that gives the information on the highest stage of the lung cancer is targeted first, thereby minimizing the need for additional procedures (15). There is evidence to show that multidisciplinary care improves the access to the most accurate staging investigations which in turn improves the accuracy of cancer staging, but this has only been reported in gastro-esophageal cancer and there is still a paucity of evidence when it comes to lung cancer $(70,71)$. In the afore-mentioned study, collective MDT staging where an overall clinical stage for each patient was determined after discussion at the MDT meeting was more accurate than any individual imaging techniques such as EUS, CT scan, and laparoscopic ultrasound for gastric and oesophageal cancer (70). Some studies have reported that thoracic MDT has resulted a more rapid turnaround time to tissue diagnosis and staging, resulting in earlier initiation of therapy (72).

In a retrospective review involving 1,222 lung cancer patients, the MDT approach resulted in an increase of subspecialty referrals by $50 \%$, leading to more rapid tissue diagnosis and staging $(73,74)$. Boxer et al. also reported that patients who were managed by a MDT had better characterization of their clinical stage, Eastern Cooperative Oncology Group performance status and type of malignancy (73). Another study also showed that patients 
discussed by an MDT were statistically more likely to have a complete staging ( $79 \%$ vs. $93 \%)$, earlier access to treatment (29 vs. 17 days) and treatment in accordance with international cancer guidelines (81\% vs. 97\%) (64). More recently, some studies have concluded that MDT management is associated with a better survival in NSCLC patients with Stage III and IV disease (75) and across all stages of disease (76-79). Patients who are discussed at an MDT meeting are more likely to receive anticancer treatment (79).

The impact of multidisciplinary care in lung cancer can be observed on certain key measures which include improved survival, accuracy of staging, access to diagnostic procedures, improvement in decision making, better utilization of resources such as radiotherapy and palliative care services, and improved care coordination, which translate into better patient experience and improved quality of life. The multidisciplinary approach also advocates standardized treatment according to guideline recommendations and encourages audit of services to improve the standards and quality of care (80). However, although recommended by professional societies, MDT management is not practiced universally.

\section{Conclusions}

Lung cancer survival and other outcomes are dependent not only on the histologic characteristics and accurate staging but also on the presence and appropriate management of comorbidities, local symptoms and treatment-related complications and toxicities. Ideally, centers managing patients with lung cancer should have MDTs with a thoracic oncology board recommending evidence-based and guideline-compliant management. Despite a relative paucity of quality evidence that the multidisciplinary care contributes to better lung cancer survival outcomes, the MDT approach has evolved to become the standard of care in many centers worldwide. Thoracic MDT has resulted in more timely investigations that lead to faster diagnosis and staging, resulting in earlier initiation of therapy. While there is evidence that access to the most accurate staging investigations is improved by multidisciplinary care which improves the accuracy of staging of gastro-esophageal cancer, there is a paucity of such evidence for lung cancer.

\section{Acknowledgments}

Funding: None.

\section{Footnote}

Provenance and Peer Review: This article was commissioned by the Guest Editor (Emily Stone) for the series "Lung Cancer Multidisciplinary Care" published in Translational Lung Cancer Research. The article was sent for external peer review organized by the Guest Editor and the editorial office.

Conflicts of Interest: All authors have completed the ICMJE uniform disclosure form (available at http://dx.doi. org/10.21037/tlcr.2019.11.28). The series "Lung Cancer Multidisciplinary Care" was commissioned by the editorial office without any funding or sponsorship. Chong-Kin Liam received research grants from AstraZeneca, Boehringer Ingelheim, and Honoraria and fees for lectures and advisory board meeting from AstraZeneca, Boehringer Ingelheim, Merck Sharp \& Dohme, Pfizer. The authors have no other conflicts of interest to declare.

Ethical Statement: The authors are accountable for all aspects of the work in ensuring that questions related to the accuracy or integrity of any part of the work are appropriately investigated and resolved.

Open Access Statement: This is an Open Access article distributed in accordance with the Creative Commons Attribution-NonCommercial-NoDerivs 4.0 International License (CC BY-NC-ND 4.0), which permits the noncommercial replication and distribution of the article with the strict proviso that no changes or edits are made and the original work is properly cited (including links to both the formal publication through the relevant DOI and the license). See: https://creativecommons.org/licenses/by-nc-nd/4.0/.

\section{References}

1. Siegel R, Naishadham D, Jemal A. Cancer statistics. CA Cancer J Clin 2013;63:11-30.

2. Silvestri GA, Littenberg B, Colice GL. The clinical evaluation for detecting metastatic lung cancer. A metaanalysis. Am J Respir Crit Care Med 1995;152:225-30.

3. Feinstein AR, Wells CK. A clinical-severity staging system for patients with lung cancer. Medicine (Baltimore) 1990;69:1-33.

4. National Comprehensive Cancer Network (NCCN). NCCN clinical practice guidelines in oncology: non-small cell lung cancer, V1.2020. [Accessed 9 November 2019.] 
Available online: http://www.nccn.org/professionals/ physician_gls/pdf/nscl.pdf

5. Rami-Porta R, editor. International Association for the Study of Lung Cancer Staging Manual in Thoracic Oncology, 2nd Edn. Editorial Rx Press, North Fort Myers, FL, 2016.

6. Ost DE, Ernst A, Lei X, et al. Diagnostic yield of endobronchial ultrasound-guided transbronchial needle aspiration: results of the AQuIRE Bronchoscopy Registry. Chest 2011;140:1557-66.

7. Bulman W, Saqi A, Powell CA. Acquisition and processing of endobronchial ultrasound-guided transbronchial needle aspiration specimens in the era of targeted lung cancer chemotherapy. Am J Respir Crit Care Med 2012;185:606-11.

8. Detterbeck FC, Lewis SZ, Diekemper R, et al. Executive summary: diagnosis and management of lung cancer, 3rd ed: American College of Chest Physicians evidence-based clinical practice guidelines. Chest 2013;143:7S-37S.

9. Travis WD, Brambilla E, Van Schil P, et al. Paradigm shifts in lung cancer as defined in the new IASLC/ATS/ ERS lung adenocarcinoma classification. Eur Respir J 2011;38:239-43.

10. Ellis PM, Blais N, Soulieres D, et al. A systematic review and Canadian consensus recommendations on the use of biomarkers in the treatment of non-small cell lung cancer. J Thorac Oncol 2011;6:1379-91.

11. Travis WD, Brambilla E, Noguchi M, et al. International association for the study of lung cancer/american thoracic society/european respiratory society international multidisciplinary classification of lung adenocarcinoma. J Thorac Oncol 2011;6:244-85.

12. Travis WD, Brambilla E, Riely GJ. New pathologic classification of lung cancer: relevance for clinical practice and clinical trials. J Clin Oncol 2013;31:992-1001.

13. Dietel M, Bubendorf L, Dingemans AM, et al. Diagnostic procedures for non-small-cell lung cancer (NSCLC): recommendations of the European Expert Group. Thorax 2016;71:177-84.

14. De Leyn P, Lardinois D, Van Schil P, et al. European trends in preoperative and intraoperative nodal staging: ESTS guidelines. J Thorac Oncol 2007;2:357-61.

15. Gaga M, Powell CA, Schraufnagel DE, et al. An official American Thoracic Society/European Respiratory Society statement: the role of the pulmonologist in the diagnosis and management of lung cancer. Am J Respir Crit Care Med 2013;188:503-7.

16. Blackhall F, Thatcher N, Booton R, et al. The impact on the multidisciplinary team of molecular profiling for personalized therapy in non-small cell lung cancer. Lung Cancer 2013;79:101-3.

17. Silvestri GA, Gonzalez AV, Jantz MA, et al. Methods for staging non-small cell lung cancer: Diagnosis and management of lung cancer, 3rd ed: American College of Chest Physicians evidence-based clinical practice guidelines. Chest 2013;143:e211S-50S.

18. Ost DE, Yeung SC, Tanoue LT, et al. Clinical and organizational factors in the initial evaluation of patients with lung cancer: Diagnosis and management of lung cancer, 3rd ed: American College of Chest Physicians evidence-based clinical practice guidelines. Chest 2013;143:e121S-41S.

19. Lindeman NI, Cagle PT, Aisner DL, et al. Updated molecular testing guideline for the selection of lung cancer patients for treatment with targeted tyrosine kinase inhibitors: guideline from the College of American Pathologists, the International Association for the Study of Lung Cancer, and the Association for Molecular Pathology. J Thorac Oncol 2018;13:323-58.

20. Travis WD, Brambilla E, Noguchi M, et al. Diagnosis of lung cancer in small biopsies and cytology: implications of the 2011 International Association for the Study of Lung Cancer/American Thoracic Society/European Respiratory Society classification. Arch Pathol Lab Med 2013;137:668-84.

21. Planchard D, Popat S, Kerr K et al. Metastatic non-small cell lung cancer: ESMO Clinical Practice Guidelines for diagnosis, treatment and follow-up. Ann Oncol 2018;29:iv192-iv237.

22. Yatabe Y, Dacic S, Borczuk AC, et al. Best practices recommendations for diagnostic immunohistochemistry in lung cancer. J Thorac Oncol 2019;14:377-407.

23. Scagliotti G, Brodowicz T, Shepherd FA, et al. Treatment by-histology interaction analyses in three phase III trials show superiority of pemetrexed in nonsquamous non-small cell lung cancer. J Thorac Oncol 2011;6:64-70.

24. Cheng $\mathrm{H}, \mathrm{Xu}$ X, Costa DB, et al. Molecular testing in lung cancer: the time is now. Curr Oncol Rep 2010;12:335-48.

25. Liam CK, Andarini S, Lee $\mathrm{P}$, et al. Lung cancer staging now and in the future. Respirology 2015;20:526-34.

26. Postmus PE, Kerr KM, Oudkerk M, et al. Early and locally advanced non-small-cell lung cancer (NSCLC): ESMO Clinical Practice Guidelines for diagnosis, treatment and follow-up. Ann Oncol 2017;28: iv1-iv21.

27. Riedel RF, Wang X, McCormack M, et al. Impact of a multidisciplinary thoracic oncology clinic on the timeliness 
of care. J Thorac Oncol 2006;1:692-6.

28. Murray PV, O'Brien ME, Sayer R, et al. The pathway study: results of a pilot feasibility study in patients suspected of having lung carcinoma investigated in a conventional chest clinic setting compared to a centralised two-stop pathway. Lung Cancer 2003;42:283-90.

29. Laroche C, Wells F, Coulden R, et al. Improving surgical resection rate in lung cancer. Thorax 1998;53:445-9.

30. Mohammed N, Kestin LL, Grills IS, et al. Rapid disease progression with delay in treatment of non-small-cell lung cancer. Int J Radiat Oncol Biol Phys 2011;79:466-72.

31. De Wever W, Ceyssens S, Mortelmans L, et al. Additional value of PET-CT in the staging of lung cancer: comparison with CT alone, PET alone and visual correlation of PET and CT. Eur Radiol 2007;17:23-32.

32. Subedi N, Scarsbrook A, Darby M, et al. The clinical impact of integrated FDG PET-CT on management decisions in patients with lung cancer. Lung Cancer 2009;64:301-7.

33. Lu Y, Xie D, Huang W, et al. 18F-FDG PET/CT in the evaluation of adrenal masses in lung cancer patients. Neoplasma 2010;57:129-34.

34. Ozcan Kara P, Kara T, Kara Gedik G, et al. The role of fluorodeoxyglucose-positron emission tomography/ computed tomography in differentiating between benign and malignant adrenal lesions. Nucl Med Commun 2011;32:106-12.

35. Grassetto G, Fornasiero A, Bonciarelli G, et al. Additional value of FDG-PET/CT in management of "solitary" liver metastases: preliminary results of a prospective multicenter study. Mol Imaging Biol 2010;12:139-44.

36. Song JW, Oh YM, Shim TS, et al. Efficacy comparison between (18)F-FDG PET/CT and bone scintigraphy in detecting bony metastases of non-small-cell lung cancer. Lung Cancer 2009;65:333-8.

37. Purandare NC, Rangarajan V, Pramesh CS, et al. Isolated asymptomatic skeletal muscle metastasis in a potentially resectable non-small cell lung cancer: detection with FDG PET-CT scanning. Cancer Imaging 2008;8:216-9.

38. Hwangbo B, Kim SK, Lee HS, et al. Application of endobronchial ultrasound-guided transbronchial needle aspiration following integrated PET/CT in mediastinal staging of potentially operable non-small cell lung cancer. Chest 2009;135:1280-7.

39. Herth FJ, Eberhardt R, Krasnik M, Ernst A. Endobronchial ultrasound-guided transbronchial needle aspiration of lymph nodes in the radiologically and positron emission tomography-normal mediastinum in patients with lung cancer. Chest 2008;133:887-91

40. Herth FJ, Ernst A, Eberhardt R, et al. Endobronchial ultrasound-guided transbronchial needle aspiration of lymph nodes in the radiologically normal mediastinum. Eur Respir J 2006;28:910-4.

41. Liao CY, Chen JH, Liang JA, et al. Meta-analysis study of lymph node staging by 18 F-FDG PET/CT scan in nonsmall cell lung cancer: comparison of TB and non-TB endemic regions. Eur J Radiol 2012;81:3518-23.

42. Kozower BD, Meyers BF, Reed CE, et al. Does positron emission tomography prevent nontherapeutic pulmonary resections for clinical stage IA lung cancer? Ann Thorac Surg 2008;85:1166-9.

43. Gould MK, Kuschner WG, Rydzak CE, et al. Test performance of positron emission tomography and computed tomography for mediastinal staging in patients with non-small-cell lung cancer: a meta-analysis. Ann Intern Med 2003;139:879-92.

44. Farrell MA, McAdams HP, Herndon JE, et al. Non-small cell lung cancer: FDG PET for nodal staging in patients with stage I disease. Radiology 2000;215:886-90.

45. Gómez-Caro A, Garcia S, Reguart N, et al. Incidence of occult mediastinal node involvement in cN0 non-smallcell lung cancer patients after negative uptake of positron emission tomography/computer tomography scan. Eur J Cardiothorac Surg 2010;37:1168-74.

46. Chiba K, Isoda M, Chiba M, et al. Significance of PET/ CT in determining actual TNM staging for patients with various lung cancers. Int Surg 2010;95:197-204.

47. Lee BE, von Haag D, Lown T, et al. Advances in positron emission tomography technology have increased the need for surgical staging in non-small cell lung cancer. J Thorac Cardiovasc Surg 2007;133:746-52.

48. Prévost A, Papathanassiou D, Jovenin N, et al. Comparison between PET(-FDG) and computed tomography in the staging of lung cancer. Consequences for operability in 94 patients. Rev Pneumol Clin 2009;65:341-9.

49. Rodríguez Fernández A, Bellón Guardia ME, Gómez Río $M$, et al. Staging of non-small cell lung cancer. Diagnosis efficacy of structural (CT) and functional (FDG-PET) imaging methods. Rev Clin Esp 2007;207:541-7.

50. Morgensztern D, Goodgame B, Baggstrom MQ, et al. The effect of FDG-PET on the stage distribution of nonsmall cell lung cancer. J Thorac Oncol 2008;3:135-9.

51. Heo EY, Yang SC, Yoo CG, et al. Impact of whole-body $18 \mathrm{~F}$-fluorodeoxyglucose positron emission tomography 
on therapeutic management of non-small cell lung cancer. Respirology 2010;15:1174-8.

52. Fischer B, Lassen U, Mortensen J, et al. Preoperative staging of lung cancer with combined PET-CT. N Engl J Med 2009;361:32-9.

53. van Tinteren H, Hoekstra OS, Smit EF, et al. Effectiveness of positron emission tomography in the preoperative assessment of patients with suspected non-small-cell lung cancer: the PLUS multicentre randomised trial. Lancet 2002;359:1388-93.

54. Davis PC, Hudgins PA, Peterman SB, et al. Diagnosis of cerebral metastases: double-dose delayed CT vs contrast-enhanced MR imaging. AJNR Am J Neuroradiol 1991;12:293-300.

55. Yokoi K, Kamiya N, Matsuguma H, et al. Detection of brain metastasis in potentially operable non-small cell lung cancer: a comparison of CT and MRI. Chest 1999;115:714-9.

56. Rusch VW, Asamura H, Watanabe H, et al. The IASLC lung cancer staging project: a proposal for a new international lymph node map in the forthcoming seventh edition of the TNM classification for lung cancer. J Thorac Oncol 2009;4:568-77.

57. Adams K, Shah PL, Edmonds L, et al. Test performance of endobronchial ultrasound and transbronchial needle aspiration biopsy for mediastinal staging in patients with lung cancer: systematic review and meta-analysis. Thorax 2009;64:757-62.

58. Annema JT, van Meerbeeck JP, Rintoul RC, et al. Mediastinoscopy vs endosonography for mediastinal nodal staging of lung cancer: a randomized trial. JAMA 2010;304:2245-52.

59. van der Heijden EH, Casal RF, Trisolini R, et al. Guideline for the acquisition and preparation of conventional and endobronchial ultrasound-guided transbronchial needle aspiration specimens for the diagnosis and molecular testing of patients with known or suspected lung cancer. Respiration 2014;88:500-17.

60. Asamura H, Chansky K, Crowley J, et al. The International Association for the Study of Lung Cancer Lung Cancer Staging Project: Proposals for the Revision of the N Descriptors in the Forthcoming 8th Edition of the TNM Classification for Lung Cancer. J Thorac Oncol 2015;10:1675-84.

61. Rami-Porta R, Bolejack V, Giroux DJ, et al. The IASLC lung cancer staging project: the new database to inform the eighth edition of the TNM classification of lung cancer. J
Thorac Oncol 2014;9:1618-24.

62. Lardinois D, De Leyn P, Van Schil P, et al. ESTS guidelines for intraoperative lymph node staging in non-small cell lung cancer. Eur J Cardiothorac Surg 2006;30:787-92.

63. Detterbeck FC, Boffa DJ, Kim AW, et al. The Eighth Edition Lung Cancer Stage Classification. Chest 2017;151:293-303.

64. Freeman RK, Van Woerkom JM, Vyverberg A, et al. The effect of a multidisciplinary thoracic malignancy conference on the treatment of patients with lung cancer. Eur J Cardiothorac Surg 2010;38:1-5.

65. Forrest LM, McMillan DC, McArdle CS, et al. An evaluation of the impact of a multidisciplinary team, in a single centre, on treatment and survival in patients with inoperable non-small-cell lung cancer. Br J Cancer 2005;93:977-8.

66. Brunelli A, Charloux A, Bolliger CT, et al. European Respiratory Society and European Society of Thoracic Surgeons Joint Task Force on Fitness for Radical Therapy. ERS/ESTS clinical guidelines on fitness for radical therapy in lung cancer patients (surgery and chemo-radiotherapy). Eur Respir J 2009;34:17-41.

67. Coory M, Gkolia P, Yang IA, et al. Systematic review of multidisciplinary teams in the management of lung cancer. Lung Cancer 2008;60:14-21.

68. Patkar V, Acosta D, Davidson T, et al. Cancer multidisciplinary team meetings: evidence, challenges and the role of clinical decision support technology. Int J Breast Cancer 2011;2011:831605.

69. Fleissig A, Jenkins V, Catt S, et al. Multidisciplinary teams in cancer care: are they effective in the UK? Lancet Oncol 2006;7:935-43.

70. Davies AR, Deans DAC, Penman I, et al. The multidisciplinary team meeting improves staging accuracy and treatment selection for gastro-esophageal cancer. Dis Esophagus 2006;19:496-503.

71. Stephens MR, Lewis WG, Brewster AE, et al. Multidisciplinary team management is associated with improved outcomes after surgery for esophageal cancer. Dis Esophagus. 2006;19:164-71.

72. Horvath LE, Yordan E, Malhotra D, et al. Multidisciplinary care in the oncology setting: historical perspective and data from lung and gynecology multidisciplinary clinics. J Oncol Pract 2010;6:e21-6.

73. Boxer MM, Vinod SK, Shafiq J, et al. Do multidisciplinary team meetings make a difference in the management of 
lung cancer? Cancer 2011;117:5112-20.

74. Lau KK, Rathinam S, Waller DA, et al. The effects of increased provision of thoracic surgical specialists on the variation in lung cancer resection rate in England. J Thorac Oncol 2013;8:68-72.

75. Pan CC, Kung PT, Wang YH, et al. Effects of multidisciplinary team care on the survival of patients with different stages of non-small cell lung cancer: a national cohort study. PLoS One 2015;10: e0126547.

76. Prades J, Remue E, van Hoof E, et al. Is it worth reorganising cancer services on the basis of multidisciplinary teams (MDTs)? A systematic review of the objectives and organisation of MDTs and their impact on patient outcomes. Health Policy 2015;119:464-74.

Cite this article as: Liam CK, Liam YS, Poh ME, Wong CK. Accuracy of lung cancer staging in the multidisciplinary team setting. Transl Lung Cancer Res 2020;9(4):1654-1666. doi: 10.21037/tlcr.2019.11.28
77. Bilfinger TV, Albano D, Perwaiz M, et al. Survival outcomes among lung cancer patients treated using a multidisciplinary team approach. Clin Lung Cancer 2018;19:346-51.

78. Stone E, Rankin N, Kerr S, et al. Does presentation at multidisciplinary team meetings improve lung cancer survival? Findings from a consecutive cohort study. Lung Cancer 2018;124:199-204.

79. Mitchell PL, Thursfield VJ, Ball DL, et al. Lung cancer in Victoria: are we making progress? Med J Aust 2013;199:674-9.

80. Denton E, Conron M. Improving outcomes in lung cancer: the value of the multidisciplinary health care team. J Multidiscip Healthc 2016;9:137-44. 